\title{
Real road networks on digital maps with applications in the search for optimal routes
}

\author{
Autores \\ Amelec Viloria, Noel Varela, David Ovallos-Gazabon, Omar Bonerge Pineda Lezama, \\ Alberto Roncallo, Jairo Martinez Ventura
}

\section{Abstract}

Google Maps web mapping service allows, through its extensive API development tool, to extract, process and store updated and real-time road information such as the aerial view of a road network, the travel time and distance between two points and the geographic coordinates of intersections (Di Natale et al. in understanding and using the controller area network communication protocol. Springer, New York, NY, 2012 [1]]). However, trivial data required in the construction of the digraph, such as the relationship of the streets associated to those intersections and the type of direction that corresponds to each street, do not exist as an attribute in the API since they are not freely accessible or an excessive cost must be paid for the database. Therefore, a practical way to obtain this specific information is through the development of an application that allows the visual selection of the characteristic elements of a network and the extraction of the necessary data in the construction of related digraphs as a tool in the solution of road problems (Rutty et al. in Transp Res Part Transp Environ 24:44-51, 2013 [2]). This research proposes a method to build digraphs with an application in the Google Maps API in the visual extraction of elements such as vertices (intersections), edges (streets) and direction arrows (road direction), allowing the application of Dijkstra's algorithm in search of alternative routes.

Palabras clave:

Digraph, Road network, Google Maps API, Dijkstra's algorithm, Alternative route 